\title{
EVT-BASED ESTIMATION OF RISK CAPITAL AND CONVERGENCE OF HIGH QUANTILES
}

\author{
MATTHIAS DEGEN *** AND \\ PAUL EMBRECHTS, ${ }^{*}$ ETH Zurich
}

\begin{abstract}
We discuss some issues regarding the accuracy of a quantile-based estimation of risk capital. In this context, extreme value theory (EVT) emerges naturally. The paper sheds some further light on the ongoing discussion concerning the use of a semi-parametric approach like EVT and the use of specific parametric models such as the $g$-and- $h$. In particular, we discusses problems and pitfalls evolving from such parametric models when using EVT and highlight the importance of the underlying second-order tail behavior.
\end{abstract}

Keywords: Extreme value theory; $g$-and- $h$ distribution; operational risk; peaks over threshold; penultimate approximation; second-order regular variation; slow variation; value at risk

2000 Mathematics Subject Classification: Primary 60G70

Secondary $62 \mathrm{G} 32$

\section{Introduction}

Over recent years, we have witnessed a growing interest in the theory and applications of extreme value theory (EVT). For instance, textbooks such as [2], [11], or [26] discuss new methodological developments together with specific applications to such fields as environmental statistics, telecommunication, insurance, and finance. In applying EVT we still run into theoretical issues which need further study. In this paper we present such a problem, discuss some partial solutions, and indicate where more research is needed.

Our starting point is a problem from the regulatory framework (the so-called Basel II) of banking and finance. The reader interested in this more applied background is referred to Chapter 1 of [23]. For the purposes of the present paper, we concentrate on the quantitative modeling of operational risk (OR). OR is defined as the risk of losses resulting from inadequate or failed internal processes, people and systems, or from external events. This definition includes legal risk, but excludes strategic and reputational risk. OR can be viewed as complementary to the widely studied risk classes of market risk and credit risk. Without going into a full description of OR data, it suffices to know that risk capital for OR has to be calculated (statistically estimated) using the concept of value at risk ( $\mathrm{VaR})$ at the extreme level of $99.9 \%$ and for losses aggregated over a one-year period; see Section 3 for a definition of VaR. Because of this, early on EVT was recognized as a canonical tool (see [24]) but also criticized for the possible instability of its output; see [13]. Degen et al. [10] highlighted some of the main problems in applying EVT to OR data and, moreover, compared and contrasted EVT with the alternative $g$-and- $h$ approach, as championed in [13]. One of the main conclusions of these earlier analyses of OR data is that in standard loss severity models used in OR practice, it is the asymptotic behavior of the tail

Received 28 February 2008; revision received 16 April 2008.

* Postal address: Department of Mathematics, ETH Zurich, Raemistrasse 101, CH-8092 Zurich, Switzerland.

** Email address: degen@math.ethz.ch 
distribution (in particular the associated slowly varying function in a Pareto-type model) that may cause problems. In the present paper we highlight these issues in more detail. In particular, we show that, for a simple distribution such as the $g$-and- $h$, EVT-based estimation for ranges relevant for practice may give answers which differ significantly from the asymptotics.

In Section 2 we give basic notation, model assumptions, and review some standard facts from EVT. In Section 3 we discuss rates of convergence for quantiles and penultimate approximations. In Section 4 we look more carefully into the second-order tail behavior under specific model assumptions for EVT applications to OR. We show that the slowly varying function underlying the $g$-and- $h$ model has second-order properties which may give rise to misleading conclusions when such data are analyzed using standard EVT methodology. We conclude in Section 5 and give hints for further research.

\section{Univariate EVT-background and notation}

We assume that the reader is familiar with univariate EVT, as presented, for instance, in [14]. Below we review some basic facts. Throughout, we assume that our loss data $X$ are modeled by a continuous distribution function (DF) $F(x)=\mathrm{P}(X \leq x)$ and we standardly write $\bar{F}=1-F$.

We use the notation $\operatorname{MDA}\left(H_{\xi}\right)$ for the maximum domain of attraction of a generalized extreme value DF $H_{\xi}$; see [14] for details. Throughout the paper, we restrict our attention to the case in which $\xi>0$. Then $F \in \operatorname{MDA}\left(H_{\xi}\right)$ is equivalent to $\bar{F} \in R V_{-1 / \xi}$, the class of regularly varying functions with index $-1 / \xi$; see, for instance, [14, Theorem 3.3.7]. In terms of its tail quantile function $U(x)=F^{\leftarrow}(1-1 / x)$, this is equivalent to $U \in R V_{\xi}$. Here $F^{\leftarrow}$ denotes the generalized inverse of $F$; see Definition 3.1, below. We standardly use the notation $\bar{F} \in R V_{-1 / \xi}$ for $\bar{F}(x)=x^{-1 / \xi} L(x)$, where $L$ is some slowly varying function in the sense of Karamata, i.e. for all $x>0$,

$$
\lim _{t \rightarrow \infty} \frac{L(t x)}{L(t)}=1 .
$$

We write $L_{F}$ and $L_{U}$ for the slowly varying functions associated with $\bar{F}$ and $U$, respectively. The functions $F$ and $U$ are always assumed to be continuous and sufficiently smooth where needed.

Often, it turns out to be more convenient to work on a log-log scale for $\bar{F}$ and $U$. As such, for $\bar{F} \in R V_{-1 / \xi}$ with density $f$, we will write

$$
\bar{F}(x)=\mathrm{e}^{-\Psi(s)}=\exp \left(-\frac{s}{\xi}+\Psi_{L_{F}}(s)\right), \quad s=\log x,
$$

where $\Psi$ and $\Psi_{L_{F}}$ denote the log-log transform of $\bar{F}$ and $L_{F}$, respectively; see Appendix A for details. Similarly, we define $U(t)=\mathrm{e}^{\varphi(r)}=\exp \left(\xi r+\varphi_{L_{U}}(r)\right)$ with $r=\log t$. Note that $L$ varies slowly if $\Psi_{L}^{\prime}$ vanishes at $\infty$.

For $F \in \operatorname{MDA}\left(H_{\xi}\right)$, the result below yields a natural approximation for the excess DF $F_{u}$, defined by $F_{u}(x)=\mathrm{P}(X-u \leq x \mid X>u)$, in terms of the generalized Pareto distribution $(G P D)$ function $G_{\xi, \beta}$, defined by $G_{\xi, \beta}(x)=1-(1+\xi x / \beta)^{-1 / \xi}$, where $1+\xi x / \beta>0$.

Proposition 2.1. (Pickands-Balkema-de Haan.) For $\xi \in \mathbb{R}$, the following statements are equivalent.

(i) $F \in \operatorname{MDA}\left(H_{\xi}\right)$. 
(ii) There exists a strictly positive measurable function $\beta$ such that

$$
\lim _{u \rightarrow x_{F}} \sup _{x \in\left(0, x_{F}-u\right)}\left|F_{u}(x)-G_{\xi, \beta(u)}(x)\right|=0 .
$$

The defining property of the scale function $\beta$ is given by the asymptotic relationship $\beta(u) \sim$ $u \xi$ as $u \rightarrow \infty$. Moreover, if (2.2) holds in the case in which $\xi>0$ for some function $\beta>0$ then it also holds for $\beta(u)=\xi u$; see [11, Theorem 1.2.5].

Remark 2.1. A key property (and, hence, data assumption) underlying EVT estimation based on Proposition 2.1 is that of stability. The class of GPDs is stable under successive conditioning over increasing thresholds, and this with the same parameter $\xi$; see [14, Theorem 3.4.13(c) and subsequent Remark 5]. All EVT-based estimation procedures are based on this tail-stability property. It may typically hold for environmental data; whether or not it is tenable for socioeconomic data is an issue of current debate.

As a consequence of Proposition 2.1, for $F \in \operatorname{MDA}\left(H_{\xi}\right)$, a natural approximation of the tail $\bar{F}(x)$ for $x \geq u$ and sufficiently large $u$ is provided by $\bar{F}(u) \bar{G}_{\xi, \beta(u)}(x-u)$. For $\xi>0$, we may, without loss of generality, take $\beta(u)=\xi u$ and, hence, consider the approximation

$$
\bar{K}(u, x):= \begin{cases}\bar{F}(x), & x<u, \\ \bar{F}(u) \bar{G}_{\xi, \xi u}(x-u)=c(u) x^{-1 / \xi}, & x \geq u,\end{cases}
$$

where $c(u)=u^{1 / \xi} \bar{F}(u)=L_{F}(u)$.

For practical purposes, in order to appreciate the goodness of the tail approximation (2.3) for $x \geq u$, it is important to quantify the rate at which $\bar{K}$ converges to $\bar{F}$, i.e. to determine the rate of convergence in (2.2)—or, equivalently, in (2.1).

In an OR context, rates of convergence for loss severity distributions are discussed in [10]. In [10] the authors focused on the so-called $g$-and- $h$ distribution recently proposed by Dutta and Perry [13] to model OR losses. Recall that a random variable $X$ is said to have a $g$-and- $h$ distribution if $X$ satisfies

$$
X=a+b \frac{\mathrm{e}^{g Z}-1}{g} \exp \left(\frac{h Z^{2}}{2}\right), \quad a, g, h \in \mathbb{R}, b>0,
$$

where $Z \sim N(0,1)$. The linear transformation parameters $a$ and $b$ are of minor importance for our analysis. Unless stated otherwise, we therefore restrict our attention to the standard case in which $a=0$ and $b=1$. This class of DFs was introduced in [28] and studied from a statistical point of view, for instance, in [18] and [22].

Degen et al. [10] showed that, for $g, h>0$ (typical for OR data), the $g$-and- $h$ distribution tail is regularly varying with index $-1 / h$, i.e. $\bar{F}(x)=x^{-1 / h} L_{F}(x)$. The corresponding slowly varying $L_{F}$ is, modulo constants, asymptotically of the form $\exp (\sqrt{\log x}) / \sqrt{\log x}$ (see also (4.2), below) and turns out to be a particularly difficult function to handle from a statistical data analysis point of view. Indeed, below we show that the behavior of $L_{F}$ in ranges relevant for practice is very different from its limit behavior, which may cause EVT-based estimation methods of $h$ to be very inaccurate over such ranges.

\section{Convergence of quantiles}

In quantitative risk management, risk capital charges are often based on estimates of high quantiles (VaR) of underlying profit-and-loss distributions; see Chapters 1 and 2 of [23] for details. 
Definition 3.1. The generalized inverse of a DF F,

$$
F^{\leftarrow}(q)=\inf \{x \in \mathbb{R}: F(x) \geq q\}, \quad 0<q<1,
$$

is called the quantile function of $F$. In a financial risk management context, for given $q, F^{\leftarrow}(q)$ is referred to as the $q 100 \% \mathrm{VaR}$, denoted by $\operatorname{VaR}_{q}(F)$.

The tail approximation, (2.3), suggests estimating the VaR of an (unknown) underlying DF $F \in \operatorname{MDA}\left(H_{\xi}\right)$, i.e. estimating $F^{\leftarrow}(q)$ for some level $q \in(0,1)$, typically close to 1 , by its approximating counterpart $K^{\leftarrow}(u, q)$. By the properties of inverse functions (see, for instance, [25, Proposition 0.1]), the quantiles $K^{\leftarrow}(\cdot, q)$ converge pointwise to the true value $F^{\leftarrow}(q)$ as $u \rightarrow \infty$, but the convergence need not be uniform.

Definition 3.2. We say that, for some DF $F \in \operatorname{MDA}\left(H_{\xi}\right)$, uniform relative quantile (URQ) convergence holds if

$$
\lim _{u \rightarrow \infty} \sup _{q \in(0,1)}\left|\frac{K^{\leftarrow}(u, q)}{F^{\leftarrow}(q)}-1\right|=0 ;
$$

see also Makarov [21], who gave a necessary condition for URQ convergence.

According to Makarov [21], failure of URQ convergence may lead to unrealistic risk capital estimates. Another possible reason for the discrepancy between an EVT-based methodology and certain parametric approaches for high quantile estimation is provided by the fact that the excess DFs of many loss models used in practice (for instance, log-normal, log-gamma, and $g$-and- $h$ ) show very slow rates of convergence to the GPD. At the model level, this is due to the second-order behavior of the underlying slowly varying functions. Consequently, tail index estimation and also quantile (i.e. risk capital) estimation using EVT-based methodology improperly may yield inaccurate results; see [10]. Below we combine both lines of reasoning and embed URQ convergence in the theory of second-order regular variation.

\subsection{Rates of convergence for quantiles}

Assume that $\bar{F} \in R V_{-1 / \xi}$ for some $\xi>0$ or, equivalently, $U \in R V_{\xi}$, in terms of its tail quantile function $U(t)=F^{\leftarrow}(1-1 / t)$. In order to assess the goodness of the tail approximation, (2.3), the rate at which $K^{\leftarrow}(u, q)$ tends to the true quantile $F^{\leftarrow}(q)$ as $u \rightarrow \infty$ has to be specified. We are thus interested in the rate at which

$$
\frac{U(t x)}{U(t)}-x^{\xi}
$$

tends to 0 as $t \rightarrow \infty$.

In the sequel we focus on the rate of convergence in (3.1) within the framework of the theory of second-order regular variation, as presented, for instance, in [11, Section 2.3 and Appendix B] or [12].

The function $U$ is said to be of second-order regular variation, written $U \in 2 R V_{\xi, \rho}, \xi>0$ and $\rho \leq 0$, if, for some positive or negative function $A$ with $\lim _{t \rightarrow \infty} A(t)=0$,

$$
\lim _{t \rightarrow \infty} \frac{U(t x) / U(t)-x^{\xi}}{A(t)}=: H_{\xi, \rho}(x), \quad x>0,
$$

exists for some $H_{\xi, \rho}$ which is nontrivial. In this case we necessarily have $H_{\xi, \rho}(x)=x^{\xi}$ ( $x^{\rho}-$ $1) / \rho$ for $x>0$. Note that, for $\xi>0, U \in 2 R V_{\xi, \rho}$ is equivalent to $\bar{F} \in 2 R V_{-1 / \xi, \rho / \xi}$. 
In order to find sufficient conditions for (3.2) to hold, consider the following intuitive reasoning in terms of $\log -\log$ transforms. Let $U \in R V_{\xi}$, and assume that $U^{\prime}$ exists. This ensures that we may write $U$ as

$$
U(t)=\mathrm{e}^{\varphi(\log t)}, \quad \varphi(r)=\int_{1}^{\mathrm{e}^{r}} \frac{\varepsilon(y)}{y} \mathrm{~d} y+c,
$$

where $\varepsilon(y)=y U^{\prime}(y) / U(y)$ and $c=\log U(1)$; see Appendix A. The log-log plot of $U$ depicts the graph of $\varphi$. With $s=\log x$ and $r=\log t$, we may write (3.1) in terms of log-log transforms as

$$
\frac{U(t x)}{x^{\xi} U(t)}-1=\mathrm{e}^{\varphi(r+s)-\varphi(r)-\xi s}-1 \sim \varphi(r+s)-\varphi(r)-\xi s, \quad r=\log t \rightarrow \infty .
$$

The expression $\varphi(r+s)-\varphi(r)-\xi s$ may be approximated by $\left(\varphi^{\prime}(r)-\xi\right) s$ and, therefore, the convergence rate of $U(t x) / U(t)-x^{\xi}$ to 0 is of the same order as the rate at which $\varphi_{L_{U}}^{\prime}(\log t)=\varphi^{\prime}(\log t)-\xi=t U^{\prime}(t) / U(t)-\xi$ tends to 0 as $t \rightarrow \infty$. This motivates the next result.

Theorem 3.1. Suppose that $U(t)=\mathrm{e}^{\varphi(\log t)}$ is twice differentiable, and let $A(t)=\varphi^{\prime}(\log t)-\xi$. If, for some $\xi>0$ and some $\rho \leq 0$,

(i) $\lim _{t \rightarrow \infty} \varphi^{\prime}(t)=\xi$,

(ii) $\varphi^{\prime}(t)-\xi$ is of constant sign near $\infty$, and

(iii) $\lim _{t \rightarrow \infty} \varphi^{\prime \prime}(t) /\left(\varphi^{\prime}(t)-\xi\right)=\rho$,

then, for $x>0$,

$$
\lim _{t \rightarrow \infty} \frac{U(t x) / U(t)-x^{\xi}}{A(t)}=H_{\xi, \rho}(x)
$$

with

$$
H_{\xi, \rho}(x)= \begin{cases}x^{\xi} \frac{x^{\rho}-1}{\rho}, & \rho<0 \\ x^{\xi} \log x, & \rho=0\end{cases}
$$

Proof. Recall the definition of $A(t)=\varphi^{\prime}(\log t)-\xi$, and observe that

$$
\lim _{t \rightarrow \infty} \frac{U(t x) / U(t)-x^{\xi}}{A(t)}=H_{\xi, \rho}(x) \Longleftrightarrow \lim _{r \rightarrow \infty} \frac{\varphi(r+s)-\varphi(r)-\xi s}{\varphi^{\prime}(r)-\xi}=\mathrm{e}^{-\xi s} H_{\xi, \rho}\left(\mathrm{e}^{s}\right),
$$

where $s=\log x$ and $r=\log t$.

By assumption (iii) we have $\lim _{t \rightarrow \infty} t A^{\prime}(t) / A(t)=\rho$, which, by the representation theorem for regularly varying functions (see, for instance, [4, Theorem 1.3.1]), guarantees that $|A| \in$ $R V_{\rho}$. In particular, $A(t x) / A(t) \rightarrow x^{\rho}$ locally uniformly as $t \rightarrow \infty$, and we obtain, for every $s \in \mathbb{R}$ as $r \rightarrow \infty$,

$$
\frac{\varphi(r+s)-\varphi(r)-\xi s}{\varphi^{\prime}(r)-\xi}=\frac{\int_{0}^{s}\left(\varphi^{\prime}(r+u)-\xi\right) \mathrm{d} u}{\varphi^{\prime}(r)-\xi} \longrightarrow \int_{0}^{s} \mathrm{e}^{\rho u} \mathrm{~d} u= \begin{cases}\frac{\mathrm{e}^{\rho s}-1}{\rho}, & \rho<0 \\ s, & \rho=0 .\end{cases}
$$

This completes the proof. 
Note that in smooth cases the rate of convergence is thus uniquely determined by the underlying slowly varying function $L_{U}$, i.e. $A(t)=\varphi_{L_{U}}^{\prime}(\log t)=t L_{U}^{\prime}(t) / L_{U}(t)$.

Remarks 3.1. (URQ convergence.) (i) Define

$$
\Psi(s)=-\log \bar{F}\left(\mathrm{e}^{s}\right) \quad \text { and } \quad \Psi^{u}(s)=-\log \bar{K}\left(u, \mathrm{e}^{s}\right)
$$

for $s \in[\log u, \infty)$ (and $\Psi \equiv \Psi^{u}$ on $(-\infty, \log u)$ by definition of $\bar{K}$ ). With this notation, URQ convergence holds for $F$ if and only if $\left\|\Psi^{\leftarrow}(s)-\left(\Psi^{u}\right)^{\leftarrow}(s)\right\|_{\infty} \rightarrow 0$ on $(-\infty, 0)$ as $u \rightarrow \infty$. It is not difficult to see that URQ convergence holds for $F$ if and only if the log-log transform $\Psi_{L_{F}}$ of $L_{F}$ satisfies $\Psi_{L_{F}}(s) \rightarrow c_{F}$ as $s \rightarrow \infty$ for some constant $c_{F} \in(0, \infty)$.

(ii) For $U$ satisfying the assumptions of Theorem 3.1 with $\rho<0$, we have $\varphi^{\prime}(\log x)-\xi \sim$ $C x^{\rho}, x \rightarrow \infty$, for some constant $C>0$, and, hence, $\varphi_{L_{U}}(t)=\varphi(t)-t \xi$ (or, equivalently, $\left.\Psi_{L_{F}}(s)=s / \xi-\Psi(s)\right)$ tends to a finite limit. In particular, a strictly negative second-order parameter $\rho$ implies URQ convergence for $U$ (or, equivalently, $F$ ). In the case in which $\rho=0$, $L_{U}$ belongs to the so-called de Haan class $\Pi$, which is a subclass of the class of the slowly varying functions; see [11, Theorem B.3.6]. For $L_{U} \in \Pi, \lim _{x \rightarrow \infty} L_{U}(x)=: L_{U}(\infty)$ exists, but $L_{U}(\infty)$ may be infinite; see [11, Corollary B.2.13].

For $\bar{F} \in R V_{-1 / \xi}$, Theorem 3.1 gives the rate at which $K^{\leftarrow}$ tends to the true quantile $F^{\leftarrow}$, or, equivalently, the rate at which the corresponding properly scaled excess quantiles $F_{u}^{\leftarrow}$ converge to $G_{\xi, 1}^{\leftarrow}$. For $A(t)=t U^{\prime}(t) / U(t)-\xi$ satisfying the conditions of Theorem 3.1, we obtain, for every $x>1$,

$$
\lim _{u \rightarrow \infty} \frac{\left|F_{u}^{\leftarrow}(1-1 / x) / \xi u-G_{\xi, 1}^{\leftarrow}(1-1 / x)\right|}{A(1 / \bar{F}(u))}=\frac{1}{\xi} H_{\xi, \rho}(x)
$$

where-for sufficiently smooth $F$ - the corresponding convergence rate satisfies

$$
A\left(\frac{1}{\bar{F}(u)}\right)=\frac{\bar{F}(u)}{u f(u)}-\xi \sim \xi^{2} \frac{u L_{F}^{\prime}(u)}{L_{F}(u)} \quad \text { as } u \rightarrow \infty .
$$

Whereas, for distributions with $\rho<0$, this convergence is rather fast; it may be very slow in the case in which $\rho=0$. The log-gamma distribution, for instance, is well known for its slow convergence properties with $A(1 / \bar{F}(u))=O(1 / \log u)$. The situation for the $g$-and- $h$ distribution is even worse, with $A(1 / \bar{F}(u))=O(1 / \sqrt{\log u})$.

Summing up, in terms of the second-order parameter $\rho$, the tail $\bar{F}$ (or, equivalently, $U$ ) will be 'better' behaved if $\rho<0$ than in the case in which $\rho=0$. In the former case the convergence in (3.2) is not 'too slow', in the sense that the rate function $|A|$ is regularly varying with index $\rho<0$, i.e. the influence of the nuisance term $L_{U}$, or, equivalently, $L_{F}$, vanishes fast enough in terms of $L_{U}$ (or $L_{F}$ ) behaving 'nicely' and tending to some positive constant (thus implying URQ convergence for $F$ ). In the case in which $\rho=0$ the rate function $|A|$ is slowly varying and, hence, the excess quantiles typically converge very slowly. However, in certain cases the slow convergence rate may be improved using the concept of penultimate approximation.

\subsection{Penultimate approximations}

So far we have been concerned with the ultimate approximation, i.e. for every $x>0$ and for large values of $t$,

$$
U(t x) \approx x^{\xi} U(t)
$$


One method to improve the rate of convergence in the above approximation goes back to the seminal work of Fisher and Tippett [15]. More recent accounts on this are found, for instance, in [7], [16], or [29].

The basic idea behind penultimate approximations is to vary the shape parameter $\xi$ as a function of the threshold $t$, i.e. to consider

$$
U(t x) \approx x^{\xi(t)} U(t)
$$

with $\xi(t) \rightarrow \xi$ for $t \rightarrow \infty$, where one hopes to improve the convergence rate by choosing $\xi(\cdot)$ in an appropriate way.

In order to illustrate how to find a feasible function $\xi(\cdot)$, consider the following. In terms of $\log$-log transforms, the ultimate approximation uses the fact that, for large values of $r$,

$$
\varphi(r+s) \approx \varphi(r)+\xi s,
$$

where $r=\log t$ and $s=\log x$, i.e. $\varphi$ is approximated linearly by a straight line with slope $\xi$. A better approximation might be achieved if, for large values of $r, \varphi$ is still approximated linearly, but now by its tangent line in the respective points $r=\log t$, i.e. by a straight line with slope $\varphi^{\prime}$, leading to

$$
\varphi(r+s) \approx \varphi(r)+\varphi^{\prime}(r) s .
$$

Thus, a reasonable choice of a threshold-dependent shape parameter is $\xi(t)=\varphi^{\prime}(\log t)=$ $t U^{\prime}(t) / U(t)$.

At this point it is worth noting that there is a close connection between the theory of penultimate approximations and the theory of second-order regular variation. Suppose that $U$ satisfies the conditions given in Theorem 3.1. Then we obtain, for large values of $t$,

$$
U(t x) \approx U(t)\left(x^{\xi}+A(t) H_{\xi, \rho}(x)\right),
$$

or, equivalently, for large values of $r=\log t$,

$$
\varphi(r+s) \approx \varphi(r)+\xi s+\left(\varphi^{\prime}(r)-\xi\right) \mathrm{e}^{-\xi s} H_{\xi, \rho}\left(\mathrm{e}^{s}\right),
$$

which, by definition of $H_{\xi, \rho}$ for $\rho=0$, is the same as $\varphi(r+s) \approx \varphi(r)+\varphi^{\prime}(r) s$. As a consequence, in the case in which $\rho=0$ the theory of second-order regular variation yields (asymptotically) the same approximation as the penultimate theory. Note, however, that we may easily construct examples where the second-order theory does not apply but the penultimate theory does.

Example 3.1. Consider $U(t)=\mathrm{e}^{\varphi(\log t)}$ with $\varphi(r)=\xi r+\varphi_{L_{U}}(r)=\xi r+\sin \sqrt{r}$. In this case $\varphi_{L_{U}}$ changes sign infinitely often as we move out, and, thus, Theorem 3.1 does not apply. A penultimate approximation $\varphi(r+s)=\varphi(r)+\varphi^{\prime}(r) s$ may nevertheless be considered. In particular, the approximation error, $e_{r}(s)=\varphi(r+s)-\varphi(r)-\varphi^{\prime}(r) s$, is of the order $O\left(s^{2}\right)$ for $s \rightarrow 0$, whereas the error in the ultimate case, $\varphi(r+s)-\varphi(r)-\xi s$, is of the order $O(s)$.

The above example shows that, although no second-order improvement exists in that particular case, the penultimate approximation may locally still lead to an improvement over the ultimate approximation. In addition, we will show below that in the case in which $\rho=0$ the rate of convergence in the penultimate approximation may indeed (asymptotically) improve compared to the ultimate approximation. 
Intuitively, it is clear that the rate at which $U(t x) / U(t)-x^{\xi(t)}$ tends to 0 is of the same order as the rate at which the linear approximations (i.e. the tangent lines in points $t$ ) approach the straight line with slope $\xi$. Hence, the speed at which the penultimate convergence rate $a(\cdot)$ tends to 0 is of the same order as the speed at which the slope $\varphi^{\prime}$ tends to its ultimate value $\xi$, which is measured by $\varphi^{\prime \prime}$. So, as a candidate for the penultimate convergence rate, we choose $a(t)=\varphi^{\prime \prime}(\log t)=t \xi^{\prime}(t)$. Theorem 3.1(iii) implies that, for this choice of $a$, the convergence rate may asymptotically be improved only in cases where $\rho=0$, and we have $a(t)=o(A(t))$ for $t \rightarrow \infty$. Indeed, under the conditions discussed above and under the additional condition

$$
\frac{\varphi^{\prime \prime \prime}(x)}{\varphi^{\prime \prime}(x)} \rightarrow 0 \quad \text { or, equivalently, } \quad \frac{t \xi^{\prime \prime}(t)}{\xi^{\prime}(t)} \rightarrow-1, \quad x=\log t \rightarrow \infty,
$$

the (improved) penultimate rate of convergence may be given as follows.

Theorem 3.2. Let $U$ satisfy the conditions of Theorem 3.1 with $\rho=0$, and define $\xi(t)=$ $t U^{\prime}(t) / U(t)=A(t)+\xi$. If $\lim _{t \rightarrow \infty} t \xi^{\prime \prime}(t) / \xi^{\prime}(t)=-1$, and if $\xi^{\prime}$ is of constant sign near $\infty$, then, for all $x>0$,

$$
\lim _{t \rightarrow \infty} \frac{U(t x) / U(t)-x^{\xi(t)}}{t A^{\prime}(t)}=J_{\xi}(x),
$$

where

$$
J_{\xi}(x)=\frac{1}{2} x^{\xi}(\log x)^{2} .
$$

Proof. Following the lines of the proof of Theorem 3.1, first note that, for $s=\log x$ and $r=\log t$,

$$
\lim _{t \rightarrow \infty} \frac{U(t x) / U(t)-x^{\xi(t)}}{t A^{\prime}(t)}=J_{\xi}(x) \Longleftrightarrow \lim _{r \rightarrow \infty} \frac{\varphi(r+s)-\varphi(r)-\varphi^{\prime}(r) s}{\varphi^{\prime \prime}(r)}=\frac{1}{2} s^{2} .
$$

For every $s \in \mathbb{R}$, we have

$$
\frac{\varphi(r+s)-\varphi(r)-\varphi^{\prime}(r) s}{\varphi^{\prime \prime}(r)}=\int_{0}^{s} \int_{0}^{y} \frac{\varphi^{\prime \prime}(r+z)}{\varphi^{\prime \prime}(r)} \mathrm{d} z \mathrm{~d} y .
$$

Moreover, by assumption, $t \xi^{\prime \prime}(t) / \xi^{\prime}(t) \rightarrow-1$ (or, equivalently, $\varphi^{\prime \prime \prime}(x) / \varphi^{\prime \prime}(x) \rightarrow 0$ ), which implies that $\left|\varphi^{\prime \prime}\right| \in R V_{0}$, which guarantees that $\varphi^{\prime \prime}(r+s) / \varphi^{\prime \prime}(r) \rightarrow 1$ locally uniformly for $r \rightarrow \infty$, and, hence, for $r \rightarrow \infty$,

$$
\int_{0}^{s} \int_{0}^{y} \frac{\varphi^{\prime \prime}(r+z)}{\varphi^{\prime \prime}(r)} \mathrm{d} z \mathrm{~d} y \rightarrow \int_{0}^{s} \int_{0}^{y} 1 \mathrm{~d} z \mathrm{~d} y=\frac{1}{2} s^{2},
$$

which completes the proof.

Remarks 3.2. (i) We want to stress again that it is important to distinguish between the secondorder theory, which is an asymptotic theory (i.e. is concerned with the limit behavior), and the penultimate theory, which is a local theory. Only for the special case in which $\rho=0$ and under certain additional conditions do the second-order theory and the penultimate theory yield asymptotically the same approximation.

(ii) From the proof of Theorem 3.2 it is clear that, although the original rate is improved asymptotically, i.e. $t A^{\prime}(t)=o(A(t))$ for $t \rightarrow \infty$, the improvement is not spectacular, as the new rate $t A^{\prime}(t)$ is again slowly varying. Nevertheless, locally the improvements may be considerable, as we show in the next paragraph. 


\subsection{Implications for practice}

To illustrate the above results, we compare the (theoretical) relative approximation error

$$
e(u):=\left|\frac{K^{\leftarrow}(u, 1-1 / x)}{F^{\leftarrow}(1-1 / x)}-1\right|
$$

for the $99.9 \%$ quantiles (confidence level required under Basel II) as a function of the threshold $u$ in the ultimate and penultimate approximations for certain frequently used OR-loss severity models. Besides the well-known Burr and log-gamma distributions (see, for instance, [14, Chapter 1]), we consider the $g$-and- $h$, the modified Champernowe, and the generalized beta distributions.

The modified Champernowne distribution was recently proposed by Buch-Larssen et al. [5] in an OR context. Its tail DF is given by

$$
\bar{F}(x)=\frac{(M+c)^{\alpha}-c^{\alpha}}{(x+c)^{\alpha}+(M+c)^{\alpha}-2 c^{\alpha}}, \quad \alpha, M, x>0, c \geq 0 ;
$$

hence, $\bar{F} \in R V_{-\alpha}$. The density of a generalized beta (GB2) distribution is given by

$$
f(x) \propto \frac{x^{a p-1}}{\left(1+(x / b)^{a}\right)^{p+q}}, \quad a, b, p, q, x>0,
$$

so that $f \in R V_{-a q-1}$; see, for instance, [13] for its use in OR.

In Figure 1 we show the approximation errors $e(\cdot)$ in percentages for the $g$-and- $h$ and the log-gamma distributions (left) and for the Burr, the modified Champernowne, and the GB2 distributions (right). To enable a qualitative comparison across different distributions, we take the thresholds as quantile levels $q$ and scale the horizontal axis by the $99.9 \%$ quantile.

In order to compare quantitatively and to check how the GPD approximation for high quantiles performs, we fix a relative error level of $\mathrm{e}^{99.9 \%}(u)=5 \%$, say, and compute the excess probabilities over the corresponding $u$ levels. In practice, in order to estimate a $99.9 \%$ quantile using the peaks over threshold (POT) method, a certain amount $N_{u}$ of data exceeding this threshold $u$ is needed (we take $u$ such that $\mathrm{e}^{99.9 \%}(u)=5 \%$ ), so as to come up with reasonable estimates. For illustrative purposes, we choose $N_{u}$ to be 100 . From this we may infer the number $n$ of data points we would expect to have to generate, in order to have $N_{u}=100$
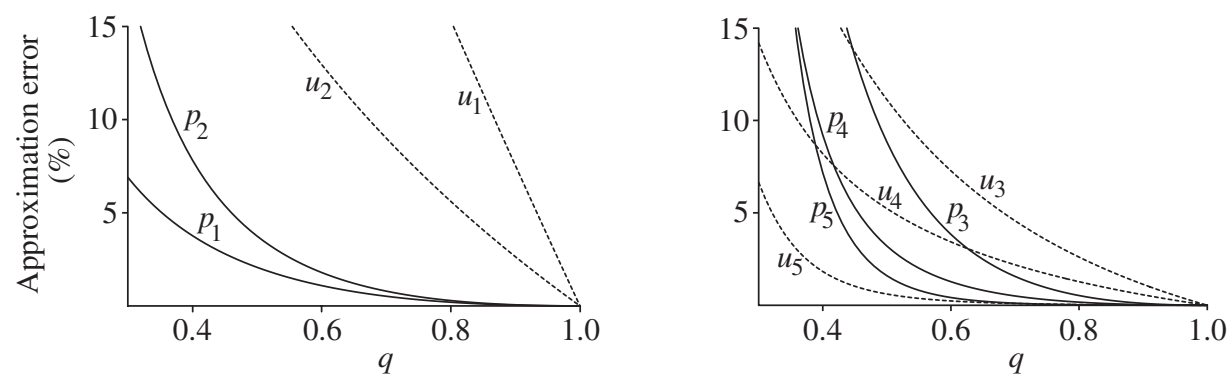

Figure 1: Approximation error for the $99.9 \%$ quantile of the $g$-and- $h$ distribution $(g=2, h=0.2$; labels $u_{1}$ and $\left.p_{1}\right)$, the log-gamma distribution $\left(\alpha=5, \beta=5\right.$; labels $u_{2}$ and $\left.p_{2}\right)$, the Burr distribution $(\alpha=3$, $\tau=1, \kappa=\frac{5}{3}$; labels $u_{3}$ and $\left.p_{3}\right)$, the modified Champernowne distribution $\left(\alpha=5, M=1, c=\frac{1}{4}\right.$; labels $u_{4}$ and $p_{4}$ ), and the GB2 distribution ( $a=5, b=1, p=1, q=1$; labels $u_{5}$ and $\left.p_{5}\right)$. 
TABLE 1: Expected number of data points $n$ needed to obtain $N_{u}=100$ exceedances over a fixed threshold $u$ for the distributions of Figure 1 with parameter values as specified in the caption.

\begin{tabular}{lcr}
\hline \multicolumn{1}{c}{ Distribution } & $\begin{array}{c}\text { Threshold } u \\
\text { such that } \mathrm{e}^{99.9 \%}(u)=5 \%\end{array}$ & \multicolumn{1}{c}{$n$} \\
\hline$g$-and- $h\left(u_{1}\right)$ & 585.00 & 91540 \\
$g$-and- $h\left(p_{1}\right)$ & 221.00 & 27740 \\
Log-gamma $\left(u_{2}\right)$ & 15.60 & 47780 \\
Log-gamma $\left(p_{2}\right)$ & 8.87 & 6260 \\
\hline Burr $\left(u_{3}\right)$ & 2.55 & 19070 \\
Burr $\left(p_{3}\right)$ & 2.13 & 9190 \\
Modified Champernowne $\left(u_{4}\right)$ & 2.41 & 4480 \\
Modified Champernowne $\left(p_{4}\right)$ & 2.15 & 2720 \\
Generalized beta $\left(u_{5}\right)$ & 1.28 & 440 \\
Generalized beta $\left(p_{5}\right)$ & 1.68 & 1440 \\
\hline
\end{tabular}

excesses over the threshold $u$ for a given relative error $\mathrm{e}^{99.9 \%}(u)=5 \%$; see Table 1 for the results.

From the five loss models considered above, the Burr, the modified Champernowne, and the GB2 satisfy $\rho<0$, whereas the log-gamma distribution and the $g$-and- $h$ distribution have second-order parameter $\rho=0$. In the latter case, $n$ increases vastly to about 47780 and 91540 for the log-gamma and the $g$-and- $h$, respectively, reflecting the slow convergence properties of these distributions.

Using penultimate approximations above a reasonably high threshold, the number of data points needed to achieve the same level of accuracy may be lowered significantly in all cases but for the GB2 distribution. Note, however, that in the GB2 case the convergence is rather fast and the approximation error $e(u)$ is negligible for high threshold values $u$ anyway. Though the theory of penultimate approximations seems to be very promising with respect to the (theoretical) improvement of high-quantile estimation accuracy, its practical relevance may be limited since the slope $\varphi^{\prime}$ has to be estimated from the data. More statistical work would be highly useful here.

Remark. The above examples are of course idealized since we assume the underlying distributions $F$ to be known and, hence, also the corresponding tail index $\xi$ of the GPD $G_{\xi}$. In practice, we will encounter an additional error source owing to estimation errors of the parameters.

From an applied risk management point of view, our analysis admits the following conclusion. The closer the second-order parameter $\rho$ is to 0, the slower EVT-based estimation techniques converge. Thus, if data seem to be modeled well by a DF $F$ with second-order parameter $\rho=0$, the amount of data needed in order to come up with reasonable results may be prohibitively large. In an OR context this is also one of the main reasons why banks have to combine internal loss data with external data and expert opinion, leading to further important statistical issues; see [20]. In addition, for distributions with 'bad' second-order behavior (i.e. $\rho=0$ ), the exact shape of the associated slowly varying function $L_{F}$ may make things worse and give rise to misleading conclusions about the underlying data. As we already saw, a prime example of this situation, important for practice, is provided by the $g$-and- $h$ slowly varying function $(g, h>0)$, which is analyzed in more detail in the next section. 


\section{A slowly varying function with a sting in its tail}

According to Dutta and Perry [13], the typical parameter values of a $g$-and- $h$ DF $F$ used to model OR-loss severities are in a range around $g \in(1.7,2.3)$ and $h \in(0.1,0.4)$. In the sequel we therefore always assume that $g$ and $h$ are strictly positive; hence, $\bar{F} \in R V_{-1 / h}$. Adopting the notation of [10], we consider $X \sim g$-and- $h$ with DF $F(x)=\Phi\left(k^{-1}(x)\right)$, where $k(x)=\left(\mathrm{e}^{g x}-1\right) / g \exp \left(h x^{2} / 2\right)$ and $\Phi$ denotes the standard normal DF. In Figure 2 we plot $\bar{F}$ on a log-log scale for OR-typical parameter values. As $\bar{F} \in R V_{-1 / h}$, a straight line with slope $-1 / h$ is to be expected as we move out in the right tail.

According to Figure 2, the log-log plot is almost linear over a large region of practical interest (for quantile levels of $90 \%$ up to $99.99 \%$ ). Therefore, over such ranges, the $g$-and- $h$ tail behavior is close to an exact Pareto and, thus, the influence of the slowly varying part seems to be minimal. Figure 2 is fallacious however, as the slope of a linear approximation $l(\cdot)$ implies a tail index of around 0.8 , whereas the theoretical tail index was chosen to be $h=0.2$. The consequences for statistical estimation of this may be better understood by the concept of what we will call local heavy tailedness.

\subsection{Local heavy tailedness}

Consider $\bar{F}(x)=x^{-1 / \xi} L_{F}(x) \in R V_{-1 / \xi}, \xi>0$, where $F$ has a density $f$. Recall that we then may write $\bar{F}$ as

$$
\bar{F}(x)=\mathrm{e}^{-\Psi(s)}=\exp \left(-\frac{s}{\xi}+\Psi_{L_{F}}(s)\right), \quad s=\log x,
$$

with $\Psi$ and $\Psi_{L_{F}}$ denoting the log-log transforms of $\bar{F}$ and $L_{F}$, respectively; see Appendix A. As a graph of $-\Psi$ corresponds to the $\log -\log$ plot of $\bar{F}$, the total amount of heavy tailedness at a point $x$ is measured by $\Psi^{\prime}(\log x)$. It consists of the ultimate heavy tailedness of the model (tail index $\xi$ ) and an additional source of local heavy tailedness owing to $L_{F}$. The local heavy tailedness is measured by the slope of $\log L_{F}$, which is given by

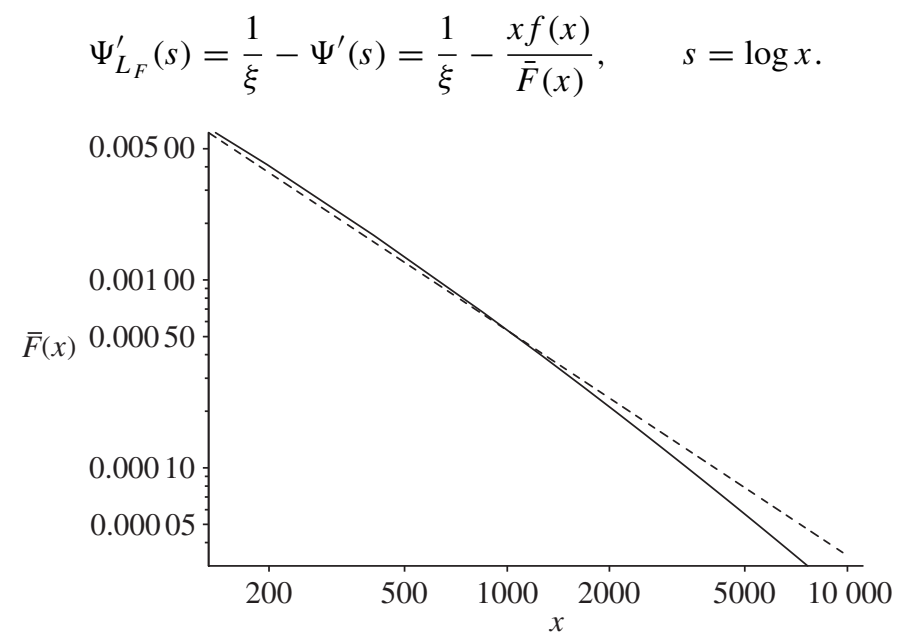

FIGURE 2: Log-log plot of the tail of a $g$-and- $h$ distribution with $g=2$ and $h=0.2$. The straight dashed line $l(\cdot)$ with slope -1.2 is plotted as a reference line. 

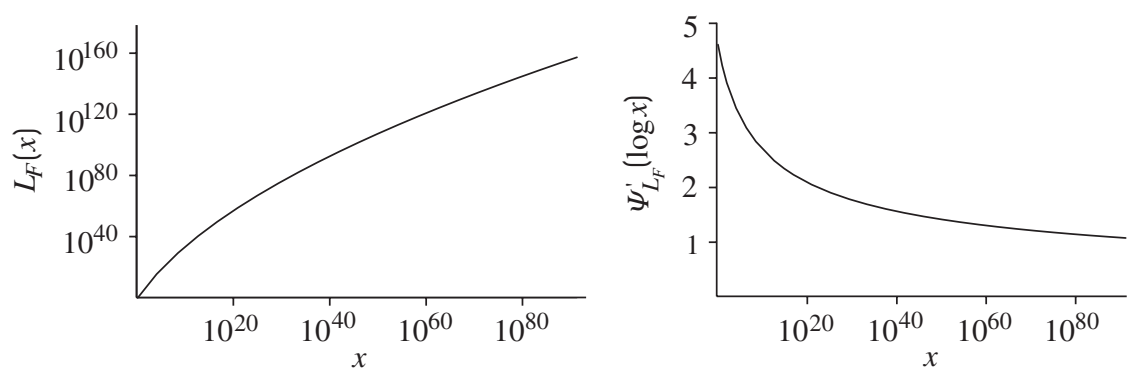

FIGURE 3: Log-log plot of the $g$-and- $h$ slowly varying function $L_{F}$ with $g=2$ and $h=0.2$ (left) with corresponding slope (right) as defined in (4.1).

Clearly, depending on the underlying model $F$, the amount of local heavy tailedness owing to the shape of its associated slowly varying function $L_{F}$ may be significant. As indicated above, this is particularly evident in the case of the $g$-and- $h$ slowly varying function, for which it turns out that the behavior of $\bar{F}$ (or $L_{F}$ ) in ranges relevant for risk management applications is very different from its ultimate asymptotic behavior. Neglecting this issue for data of (or close to) $g$-and- $h$ type may lead to problems when applying standard EVT methodology. Whereas this issue is well known from a theoretical point of view within the EVT community (see, for instance, [25, Exercise 2.4.7]), it is somewhat surprising that it manifests itself so clearly in a fairly straightforward, and increasingly used, parametric model such as the $g$-and- $h$ model.

To get a feeling for the behavior of the $g$-and- $h$ slowly varying function $L_{F}$, we show in Figure 3 a log-log plot of $L_{F}$ (left) with corresponding slope (right) for $g$ and $h$ parameter values typical for OR (the function $k$ is inverted numerically using a Newton algorithm with an error tolerance level of $10^{-13}$ ).

The almost linear log-log plot suggests that the function $L_{F}$ behaves approximately like a power function $x^{1 / \eta}$ for some $\eta>0$ and with $1 / \eta$ given in the slope plot. The asymptotic behavior of the log-log transform of $\bar{F}$ is given by

$$
-\Psi(s)=-\frac{1}{h} s+\frac{\sqrt{2} g}{h^{3 / 2}} \sqrt{s}-\frac{1}{2} \log s-c+O\left(\frac{1}{\sqrt{s}}\right), \quad s=\log x \rightarrow \infty,
$$

with $c=\frac{1}{2} \log 4 \pi / h+g^{2} / h^{2}+\log g / h$; see (A.1) in Appendix A.

The deviation from the exact power-law decay (i.e. the deviation from linearity in a log-log plot) is due to the slowly varying part. The amount of local heavy tailedness is measured by the slope $\Psi_{L_{F}}^{\prime}$ of $\log L_{F}$, which behaves like

$$
\Psi_{L_{F}}^{\prime}(s)=\frac{g}{\sqrt{2} h^{3 / 2}} \frac{1}{\sqrt{s}}-\frac{1}{2 s}+O\left(\frac{1}{s^{3 / 2}}\right), \quad s=\log x \rightarrow \infty .
$$

Therefore, the rate at which the influence of $L_{F}$ vanishes, i.e. the rate at which $\Psi_{L_{F}}^{\prime}$ tends to 0 , is of the order $O(1 / \sqrt{\log x}), x \rightarrow \infty$.

Equation (4.3) gives us a first impression of how unpleasant the $g$-and- $h$ slowly varying function might be. Indeed, its slow convergence properties together with its (power-like) behavior in ranges relevant for OR practice may lead to serious difficulties in the statistical estimation of extremes based on EVT, given that the data follow such a model. At this point we wish to stress that this is not a weak point of EVT but should rather be viewed as a warning 
against 'gormless guessing' of a parametric model, in the words of Richard Smith and Jonathan Tawn; see [14, Preface, p. VII]. In the next paragraph we study EVT estimation within a $g$-and- $h$ model, i.e. for $g$-and- $h$ generated data.

\subsection{Tail index estimation for $g$-and- $h$ data}

In [10] the problem of the estimation of the tail index $h$ within a $g$-and- $h$ model was pointed out using the Hill estimator. To emphasize that EVT-based tail index estimation for $g$-and- $h$ data may be problematic whatever method one uses, we will work below with the increasingly popular POT-MLE (maximum likelihood estimate) method for which the statistical basis was laid in the fundamental papers of Davison [8], Davison and Smith [9], and Smith [27]. For further background reading, see [14]. We additionally implemented other tail index estimators such as the moment estimator, a bias-reduced MLE, and an estimator based on an exponential regression model as discussed in [3]. As might be expected from the discussion in the previous paragraphs, all these estimators led to similar conclusions and, therefore, we refrain from showing those results.

In Figure 4, based on $n=10^{4}$ observations from a $g$-and- $h$ model ( $\left.g=2, h=0.2\right)$, we plot the POT-MLE of the tail index $h$ as a function of the number of exceedances used, together with the $95 \%$ confidence bounds.

At first glance, Figure 4 suggests that the DF of the underlying data follows nearly perfectly an exact Pareto law, or at least converges rather fast towards an exact Pareto law. Indeed, the deviation from the exact power-law behavior seems to vanish quickly as the MLE behaves stably and is flat over a large region of thresholds (as was of course to be expected from Figure 2). As a consequence, we would accept an estimate of the tail index of around $\hat{h} \approx 0.85$ (compare this value to the 0.8 implied by Figure 2 ). Thus, EVT-based estimation significantly overestimates the true parameter $h=0.2$, suggesting a rather heavy-tailed model $(\hat{\alpha}=1 / \hat{h} \approx 1.2$, i.e. infinite variance), whereas the data are simulated from a model with $1 / h=5$, which has finite moments up to order five. The reason behind this lies in the behavior of the underlying slowly varying function over moderate ranges. More precisely, for $g$-and- $h$ data $(g=2, h=0.2)$ with for practice reasonable sample sizes, a number of $k=500$, say, largest order statistics is taken into account for the estimation of $h$. For this realistic choice of parameter values of $g$ and $h$, the values of such order statistics will typically range from around 10 to around $10^{3}$. Over

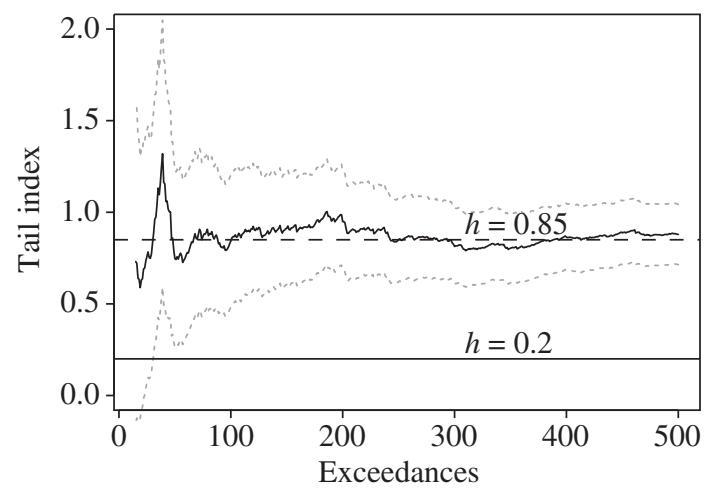

FIgURE 4: Theoretical tail index (straight solid line) and POT-MLEs of $\xi=h$ for $g$-and- $h$ data with $g=2$ and $h=0.2$, based on $n=10^{4}$ observations. 


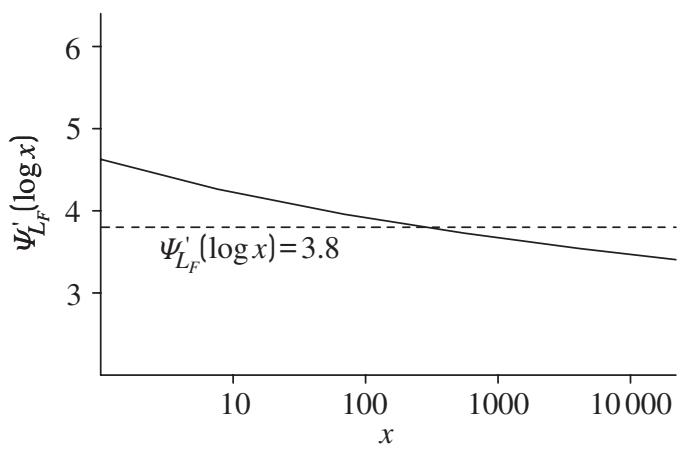

FIGURE 5: Slope of the log-log plot of the $g$-and- $h$ slowly varying function $(g=2, h=0.2)$ in a range relevant for OR.

such ranges the slope of $\Psi_{L_{F}}$ is nearly constant and, hence, $L_{F}$ grows approximately like a power $x^{1 / \eta}$ with (averaged) $1 / \eta \approx 3.8$; see Figure 5. Therefore, in ranges relevant for practical applications, the 'regularly varying'-like slowly varying part $L_{F}$ adds a significant amount of local heavy tailedness to the model. Together with the asymptotic tail decay of $\bar{F} \in R V_{-5}$, the local power-like growth of $L_{F}$ leads to a regular variation index of around -1.2, i.e. to a 'local' tail index $h_{\text {loc }}$ of $\bar{F}$ of around 0.83 .

Remark 4.1. The notion of regular variation is an asymptotic concept, hence, our emphasis on the distinction between local (i.e. finite ranges relevant for practice) and asymptotic tail behavior. Owing to its slow convergence properties, the (asymptotic) tail index for the $g$-and- $h$ distribution is significantly overestimated by standard (i.e. ultimate) EVT estimation methods. On the other hand, after a penultimate correction accounting for the local heavy tailedness owing to the behavior of the slowly varying part, the behavior of $\bar{F}$ over ranges relevant for practice is well captured by EVT methods (the MLE in Figure 4 is rather stable and flat). This is due to the extremely slow decay of $\Psi_{L_{F}}^{\prime}$, which is close to constant over large ranges. Over such ranges, the $g$-and- $h$ distribution therefore behaves approximately like an exact Pareto, for which EVT-based estimation methods are known to perform well.

Furthermore, as can be seen from (4.3), the accuracy of tail index estimation crucially depends on the ratio $g / h^{3 / 2}$ of the $g$-and- $h$ parameters. For parameter values of $g$ and $h$ relevant for OR applications (see [13, p. 43]), $g / h^{3 / 2}$ is relatively large and, thus, the slope of $\Psi_{L_{F}}$ - the share of additional local heavy tailedness-is large.

For cases with $g / h^{3 / 2}$ small, for instance, $g=0.1$ and $h=0.5, \Psi_{L_{F}}^{\prime}$ is less than 0.2 over a large range, which includes a major part of largest order statistics of reasonable sample sizes, and, thus, the influence of $L_{F}$ is minor, i.e. the difference between local and asymptotic behaviors is marginal. Consequently, the accuracy of EVT-based tail index estimation increases considerably and the estimate captures the structure of the data correctly; see, for instance, Figure 6.

\subsection{Risk capital estimation for $O R$}

Based on the 2004 loss data collection exercise (LDCE) for OR data, Dutta and Perry [13] noted that in the estimation of OR risk capital (VaR, 99.9\%, one year) there seems to be a serious discrepancy between an EVT-based approach and the $g$-and- $h$ approach. They found 


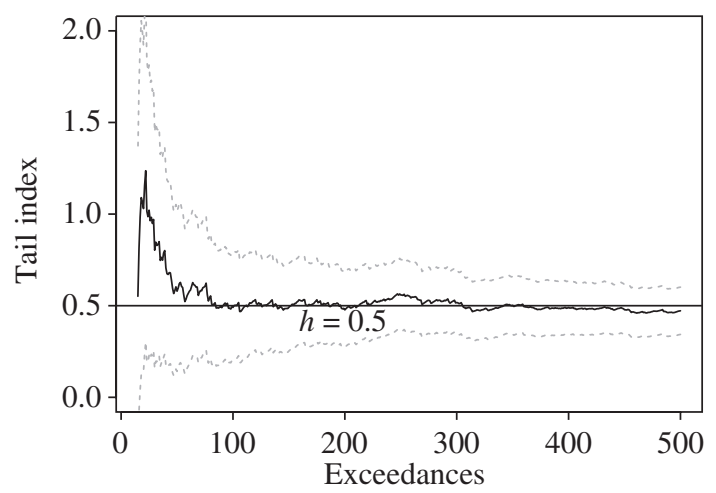

FIGURE 6: Theoretical tail index (straight solid line) and POT-MLEs of $\xi=h$ for $g$-and- $h$ data with $g=0.1$ and $h=0.5$, based on $n=10^{4}$ observations.

that EVT often yields unreasonably high risk capital estimates whereas the $g$-and- $h$ approach does not. Fitting a $g$-and- $h$ model to the 2004 LDCE data (aggregated at enterprise level), Dutta and Perry [13] found that $\xi=h \in(0.1,0.4)$ for different banks. By contrast, applying EVT methodology to the 2002 LDCE data (aggregated at business line level), Moscadelli [24] came up with infinite-mean models (i.e. $\xi>1$ ) for six out of eight business lines. Based on this, resulting risk capital estimates may be expected to differ widely for the two approaches. See also [19] on this issue; in [19] it is claimed that, for typical OR data, the $g$-and- $h$ approach is superior only from a confidence level of $99.99 \%$ onwards.

As we do not possess the data underlying these analyses, our findings below, based on simulated data, may be academic in nature. However, even though EVT-based techniques may, for simulated $g$-and- $h$ data, lead to completely wrong estimates of the (asymptotic) tail index $h$ (see Figure 4), this does not need to carry over to estimates of relevant risk measures such as VaR or return periods. Indeed, based on $n=10^{4}$ simulated $g$-and- $h$ data $(g=2, h=0.2)$, the POT-MLEs of the $99.9 \%$ quantile seem to be rather accurate (though the $95 \%$ confidence band is quite broad); see Figure 7.

The apparent incompatibility of Figures 4 and 7 can be explained by taking into account the effect of local heavy tailedness caused by the slowly varying part. For $g=2$ and $h=0.2$, the theoretical $99.9 \% g$-and- $h$ quantile is approximately 626 . The amount of local heavy tailedness in this point is $\Psi_{L_{F}}^{\prime}(626) \approx 3.7$. Moreover, recall that over large ranges the $g$-and- $h$ distribution behaves approximately like an exact Pareto distribution (with tail index $\neq h$ ), and, thus, the situation is as if we were to estimate the $99.9 \%$ quantile of a distribution which seems to be modeled well by an exact Pareto distribution with parameter $\alpha=-5+3.7=-1.3$ (i.e. with tail index $\xi \approx 0.77$ ). This quantile is then estimated using $k=500$, say, upper order statistics, whose values will-for a sample of $n=10^{4}$ observations-typically range from around 10 to around $10^{3}$. Owing to the almost constant slope of $\Psi_{L_{F}}$ (approximately equal to 3.8) over this range, the 500 order statistics thus seem to be modeled well by an exact Pareto distribution with parameter $\alpha=-1.2$ (i.e. $\xi \approx 0.83$ ). This may explain the good performance of the POT-MLEs of the $99.9 \%$ quantile in Figure 7.

Clearly, these quantile estimates are likely to get worse in cases where we are estimating far out-of-sample quantiles. Especially in an OR context, estimating at a level of $99.9 \%$ is a serious issue as today's OR-loss databases are rather sparse. But still, as the influence of $L_{F}$ changes 


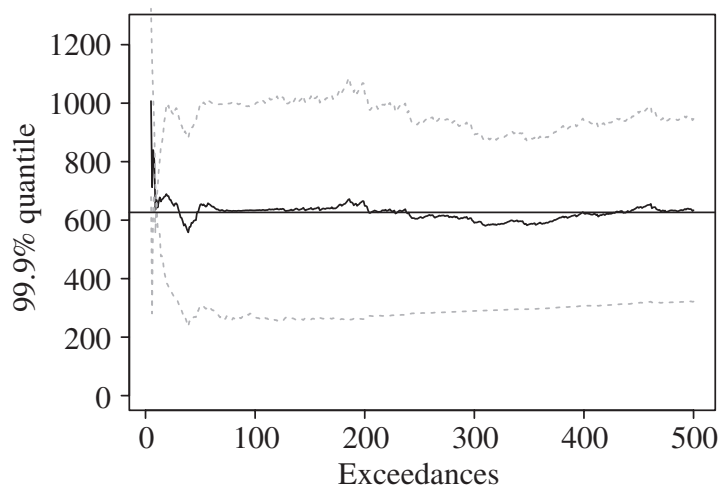

FIGURE 7: Theoretical 99.9\% $g$-and- $h$ quantile (straight solid line) and POT-MLEs for $g=2$ and $h=0.2$, based on $n=10^{4}$ observations.

only very slowly over large ranges (we are in the $\rho=0$ case), the size of estimation errors is not nearly as large as in the case for tail index estimation. In this sense, the $\rho=0$ case for high quantile estimation based on EVT is not necessarily as troublesome as can be expected from the well-known poor performance of tail index estimation in such a case. A more in-depth study of this phenomenon would however be highly desirable and have important consequences for quantitative risk management practice.

\section{Conclusion}

In this paper we have highlighted some issues regarding a quantile-based estimation of risk capital motivated by the Basel II regulatory framework for OR. From a theoretical point of view, EVT-based estimation methodologies of high quantiles arise very naturally. Our main results are as follows.

First, according to [21], failure of URQ convergence may lead to inaccurate risk capital estimates. We complement these findings by showing that, for sufficiently smooth $\bar{F} \in$ $R V_{-1 / \xi}, \xi>0$, the asymptotic behavior of the associated slowly varying function $L_{F}$ determines whether or not URQ convergence holds. This then allows us to embed URQ convergence in the framework of second-order regular variation for quantiles; $L_{F}(x) \rightarrow c \in(0, \infty)$ implies a second-order parameter $\rho<0$, whereas $L_{F}(x) \rightarrow \infty$ (or 0 ) implies that $\rho=0$. In the latter case, the slow convergence properties together with the possibly delusive behavior of $L_{F}$ may cause serious problems when applying standard EVT methodology.

Second, we stressed the fact that, when using EVT methodology, the second-order behavior of the underlying distribution, which (in smooth cases) is fully governed by its associated slowly varying function, is crucial. If data are well modeled by a distribution with 'bad' secondorder behavior, i.e. with second-order parameter $\rho=0$, EVT-based estimation techniques will typically converge slowly. As a consequence, the amount of data needed in order for EVT to deliver reasonable results may be unrealistically high and largely incompatible with today's situation for OR databases. The idea of penultimate approximations seems very promising in this respect. So far this concept has been of a more theoretical nature and further applied research would be desirable. 
Third, the $g$-and- $h$ distribution of Dutta and Perry [13] corresponds to a class of loss DFs for which the slowly varying function $L_{F}(g, h>0)$ is particularly difficult to handle. Owing to its slow convergence properties $(\rho=0)$, its behavior in ranges relevant for OR practice is very different from its ultimate asymptotic behavior. For broad ranges of the underlying loss values, the slowly varying function $L_{F}$ behaves like a regularly varying function, putting locally some extra weight to the tail $\bar{F}$. As a consequence, standard EVT-based tail index estimation (asymptotic behavior matters) may result in completely wrong estimates. However, this poor performance need not carry over to high-quantile estimation (finite-range behavior matters).

For risk management applications in general and OR in particular, a key property to look for is the second-order behavior of the underlying loss severity models. Models encountered in practice often correspond to the case in which $\rho=0$. Especially in the latter case, more research on the statistical estimation of high quantiles using EVT is needed; see, for instance, [17] and the references therein for some ideas.

Finally, as already discussed in Remark 2.1, EVT assumes certain tail-stability properties of the underlying loss data. These may or may not hold. In various fields of application outside of finance and economics these properties seem tenable and, hence, EVT has established itself as a most useful statistical modeling tool. Within financial risk management, discussions on stability are still ongoing and may lie at the basis of critical statements on the use of EVT; see, for instance, [6], [13], and [19]. In their discussion on the forecasting of extreme events, Christoffersen et al. [6] stated:

Thus, we believe that best-practice applications of EVT to financial risk management will benefit from awareness of its limitations, as well as the strengths. When the smoke clears, the contribution of EVT remains basic and useful: it helps us to draw smooth curves through the extreme tails of empirical survival functions in a way that is consistent with powerful theory. Our point is simply that we should not ask more of the theory than it can deliver.

We very much hope that our paper has helped in lifting a bit of the smokescreen and will challenge EVT experts to consider in more detail some of the statistical challenges related to the modeling of extremes in financial risk management.

\section{Appendix A}

By our standing assumption, $F$ and $F \leftarrow$ are throughout assumed to be continuous. Furthermore, we will always assume sufficient smoothness for $F$ and $F^{\leftarrow}$ where necessary. Consider $\bar{F}(x)=x^{-1 / \xi} L_{F}(x) \in R V_{-1 / \xi}, \xi>0$, and denote by $f$ the density of $F$. This is equivalent to assuming $\bar{F}$ to be normalized regularly varying with index $-1 / \xi$ (see [4, Section 1.3]), and ensures that we may write $\bar{F}$ as

$$
\bar{F}(x)=\mathrm{e}^{-\Psi(\log x)}, \quad \Psi(s)=\int_{1}^{\mathrm{e}^{s}} \frac{\eta(y)}{y} \mathrm{~d} y-c,
$$

with $\eta(y)=y f(y) / \bar{F}(y)$ and $c=\log \bar{F}(1)$. So the graph of $-\Psi$ corresponds to the $\log \log$ plot of $\bar{F}$, and we have $\Psi^{\prime}(s)=x f(x) / \bar{F}(x)$, where $s=\log x$. As $\bar{F} \in R V_{-1 / \xi}$, the slope $\Psi^{\prime}(s)$ converges to $1 / \xi$ as $s \rightarrow \infty$. The log-log transform of the associated slowly varying function $L_{F}$ is given by $\Psi_{L_{F}}(s)=s / \xi-\Psi(s)$. Its slope $\Psi_{L_{F}}^{\prime}$ tends to 0 and measures the speed at which the influence of the slowly varying nuisance part vanishes. 
Analogously, for the tail quantile function $U(t)=F^{\leftarrow}(1-1 / t)$, we obtain $U(t)=$ $\mathrm{e}^{\Psi^{\leftarrow}(\log t)}$, where $\Psi^{\leftarrow}$ denotes the generalized inverse of $\Psi$ (see Definition 3.1), and we write

$$
U(t)=\mathrm{e}^{\varphi(\log t)}, \quad \varphi(r)=\Psi^{\leftarrow}(r)=\int_{1}^{\mathrm{e}^{r}} \frac{\varepsilon(y)}{y} \mathrm{~d} y+c,
$$

with $\varepsilon(y)=y U^{\prime}(y) / U(y)$ and $c=\log U(1)$.

As $\varphi(\Psi(r))=r$, simple calculus shows that the sufficient first- and second-order conditions in the ultimate and penultimate approximations (see Theorems 3.1 and 3.2) may be equivalently expressed in terms of $\bar{F}$ or $U$.

\section{A.1. Asymptotics for the $\boldsymbol{g}$-and- $h$ distribution}

A random variable $X$ is said to have a $g$-and-h distribution if $X$ satisfies

$$
X=a+b k(Z)=a+b \frac{\mathrm{e}^{g Z}-1}{g} \exp \left(\frac{h Z^{2}}{2}\right), \quad a, g, h \in \mathbb{R}, b>0,
$$

where $Z \sim N(0,1)$. We concentrate on the case in which $a=0$ and $b=1$. Degen et al. [10] showed that, for $g, h>0$, the $g$-and- $h$ distribution is regularly varying with index $-1 / h$, i.e. $\bar{F}(x)=\bar{\Phi}\left(k^{-1}(x)\right)=x^{-1 / h} L_{F}(x)$. Since $F$ is differentiable, we may write

$$
\bar{F}(x)=\bar{\Phi}\left(k^{-1}(x)\right)=\mathrm{e}^{-\Psi(\log x)},
$$

where, as above, $\Psi$ denotes the log-log transform of $\bar{F}$.

By the definition of $k$ we have $y:=\log k(s)=h s^{2} / 2+g s-\log g+O\left(\mathrm{e}^{-g s}\right), s \rightarrow \infty$. We rewrite this as $s^{2}=2 y / h-2 g s / h+2 \log g / h+O\left(\mathrm{e}^{-g s}\right), s \rightarrow \infty$, and, hence,

$$
s=k^{-1}\left(\mathrm{e}^{y}\right)=\sqrt{\frac{2 y}{h}}-\frac{g}{h}+\frac{1}{\sqrt{y}}\left(\frac{g^{2}}{(2 h)^{3 / 2}}+\frac{\log g}{\sqrt{2 h}}\right)+O\left(\frac{1}{y^{3 / 2}}\right) \quad \text { as } y \rightarrow \infty .
$$

Recall the standard asymptotic expansion for the normal distribution tail given by $\bar{\Phi}(x)=$ $\exp \left(-x^{2} / 2\right) / \sqrt{2 \pi} x\left(1-1 / x^{2}+O\left(1 / x^{4}\right)\right), x \rightarrow \infty$; see [1, p. 932]. Hence, we obtain the following asymptotics for the $g$-and- $h$ tail $\bar{F}$ :

$$
\begin{aligned}
-\Psi(t) & =\log \bar{\Phi}\left(k^{-1}\left(\mathrm{e}^{t}\right)\right) \\
& =-\frac{1}{2}\left(k^{-1}\left(\mathrm{e}^{t}\right)\right)^{2}-\log k^{-1}\left(\mathrm{e}^{t}\right)-\log 2 \pi+O\left(\frac{1}{\left(k^{-1}\left(\mathrm{e}^{t}\right)\right)^{2}}\right) \quad \text { as } t \rightarrow \infty,
\end{aligned}
$$

which then leads to

$$
-\Psi(t)=-\frac{1}{h} t+\frac{\sqrt{2} g}{h^{3 / 2}} \sqrt{t}-\frac{1}{2} \log t-c+O\left(\frac{1}{\sqrt{t}}\right) \quad \text { as } t \rightarrow \infty,
$$

with $c=\frac{1}{2} \log 4 \pi / h+g^{2} / h^{2}+\log g / h$.

\section{A.2. Asymptotics for the log-gamma distribution}

A random variable $X$ follows a log-gamma distribution with parameters $\alpha$ and $\beta$ if its density satisfies

$$
f(x)=\frac{\alpha^{\beta}}{\Gamma(\beta)}(\log x)^{\beta-1} x^{-\alpha-1}, \quad \alpha, \beta>0, x>1,
$$


where $\Gamma$ denotes the gamma function. The corresponding tail is given by

$$
\bar{F}(x)=\frac{\Gamma(\beta, \alpha \log x)}{\Gamma(\beta)}=\mathrm{e}^{-\Psi(\log x)}, \quad x>1,
$$

where

$$
\Gamma(\beta, \alpha \log x)=\int_{\alpha \log x}^{\infty} t^{\beta-1} \mathrm{e}^{-t} \mathrm{~d} t, \quad x>1,
$$

denotes the upper incomplete gamma function. Therefore, we obtain $-\Psi(t)=\log \Gamma(\beta, \alpha t)-$ $\log \Gamma(\beta)$. For $\beta=1$, we are in the Pareto case with $-\Psi(t)=-\alpha t$. For $\beta \in \mathbb{R}_{+} \backslash\{1\}$, using the standard asymptotic expansion for the upper incomplete gamma function given in [1, p. 263], we obtain

$$
-\Psi(t)=-\alpha t+(\beta-1) \log t+c+O\left(\frac{1}{t}\right) \quad \text { as } t \rightarrow \infty,
$$

where $c=(\beta-1) \log \alpha-\log \Gamma(\beta)$.

\section{Acknowledgements}

The authors would like to thank Guus Balkema for many valuable comments and suggestions that considerably helped to improve this paper and Johanna Nešlehová for early discussions related to the project of uniform quantile convergence. An anonymous referee made several useful remarks.

\section{References}

[1] Abramowitz, M. And Stegun, I. A. (1972). Handbook of Mathematical Functions. Dover, New York.

[2] Balkema, G. And Embrechts, P. (2007). High Risk Scenarios and Extremes - A Geometric Approach. EMS, Zürich.

[3] Beirlant, J., Goegebeur, Y., Segers, J. and Teugels, J. (2004). Statistics of Extremes. John Wiley, Chichester.

[4] Bingham, N. H., Goldie, C. M. And Teugels, J. L. (1987). Regular Variation. Cambridge University Press.

[5] Buch-Larssen, T., Nielsen, J. P., Guillén, M. and Bolancé, C. (2005). Kernel density estimation for heavytailed distributions using the Champernowne transformation. Statistics 39, 503-518.

[6] Christoffersen, P. F., Diebold, F. X. and Schuermann, T. (1998). Horizon problems and extreme events in financial risk management. In Economic Policy Review, Federal Reserve Bank of New York, pp. 109-118.

[7] Cohen, J. P. (1982). Convergence rates for the ultimate and penultimate approximations in extreme-value theory. Adv. Appl. Prob. 14, 833-854.

[8] Davison, A. C. (1984). Modelling excesses over high thresholds, with an application. In Statistical Extremes and Applications, ed. J. Tiego de Oliveira, Reidel, Dordrecht, pp. 461-482.

[9] Davison, A. C. And Smith, R. L. (1990). Models for exceedances over high thresholds (with discussion). J. R. Statist. Soc. B 52, 393-442.

[10] Degen, M., Embrechts, P. And Lambrigger, D. D. (2007). The quantitative modeling of operational risk between $g$-and- $h$ and EVT. ASTIN Bull. 37, 265-291.

[11] De HaAn, L. and Ferreira, A. (2006). Extreme Value Theory - An Introduction. Springer, New York.

[12] De Haan, L. and Stadtmüller, U. (1996). Generalized regular variation of second order. J. Austral. Math. Soc. 61, 381-395.

[13] Dutta, K. And Perry, J. (2006). A tale of tails: an empirical analysis of loss distribution models for estimating operational risk capital. Working Paper 06-13, Federal Reserve Bank of Boston.

[14] Embrechts, P., Klüppelberg, C. And Mikosch, T. (1997). Modelling Extremal Events for Insurance and Finance. Springer, Berlin.

[15] Fisher, R. A. And Tippett, L. H. T. (1928). Limiting forms of the frequency distribution of the largest or smallest member of a sample. Proc. Camb. Philos. Soc. 24, 180-190.

[16] Gomes, M. I. AND DE HAAN, L. (1999). Approximation by penultimate extreme value distributions. Extremes 2, 71-85.

[17] Gomes, M. I. and Pestana, D. (2007). A sturdy reduced-bias extreme quantile (VaR) estimator. J. Amer. Statist. Assoc. 102, 280-292. 
[18] Hoaglin, D. C., Mosteller, F. And Tukey, J. W. (1985). Exploring Data Tables, Trends, and Shapes. John Wiley, New York.

[19] JoвST, A. A. (2007). Operational risk: the sting is still in the tail but the poison depends on the dose. J. Operational Risk 2, 3-59.

[20] Lambrigger, D. D., Shevchenko, P. And Wüthrich, M. (2007). The quantification of operational risk using internal data, relevant external data and expert opinions. J. Operational Risk 2, 3-27.

[21] Makarov, M. (2006). Extreme value theory and high quantile convergence. J. Operational Risk 1, 51-57.

[22] Martinez, J. And Iglewicz, B. (1984). Some properties of the Tukey $g$ and $h$ family of distributions. Commun. Statist. Theory Meth. 13, 353-369.

[23] McNeil, A. J., Frey, R. And Embrechts, P. (2005). Quantitative Risk Management: Concepts, Techniques and Tools. Princeton University Press.

[24] Moscadelli, M. (2004). The modelling of operational risk: experience with the analysis of the data collected by the Basel Committee. Working Paper 517, Bank of Italy.

[25] Resnick, S. I. (1987). Extreme Values, Regular Variation and Point Processes. Springer, New York.

[26] Resnick, S. I. (2007). Heavy-Tail Phenomena: Probabilistic and Statistical Modeling. Springer, New York.

[27] Sмiтh, R. L. (1987). Estimating tails of probability distributions. Ann. Statist. 15, 1174-1207.

[28] Tukey, J. W. (1977). Exploratory Data Analysis. Addison-Wesley, Reading, MA.

[29] Worms, R. (2002). Penultimate approximation for the distribution of the excesses. ESAIM Prob. Statist. 6, 21-31. 